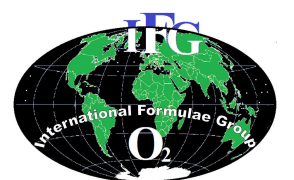

Available online at http://www.ifgdg.org

Int. J. Biol. Chem. Sci. 10(4): 1626-1636, August 2016

International Joumal

of Biological and

Chemical Sciences

ISSN 1997-342X (Online), ISSN 1991-8631 (Print)

Original Paper http://ajol.info/index.php/ijbcs http://indexmedicus.afro.who.int

\title{
Les fourmis (Hymenoptera, Formicidae) de l'enclos d'acclimatation de Katané de la réserve de faune du Ferlo nord (Sénégal)
}

\author{
M. M. DIENG ${ }^{1}$, A. B. NDIAYE ${ }^{2 *}$, Ch. T. BA ${ }^{1}$ et B. TAYLOR ${ }^{3}$ \\ ${ }^{I}$ Département de Biologie Animale, Faculté des Sciences et Techniques, UCAD, Dakar, Sénégal. \\ ${ }^{2}$ Laboratoire de Zoologie des Invertébrés Terrestres, IFAN, UCAD, B. P. 206, Sénégal. \\ ${ }^{3} 11$, Grazingfield, Wilford, Nottingham, NG11 7FN, U.K. \\ *Corresponding author; E-mail: abdoulayeb.ndiaye@ucad.edu.sn
}

\section{RESUME}

Les fourmis (Hymenoptera, Formicidae), de par leur biomasse, et leur diversité spécifique, jouent un rôle fondamental dans les écosystèmes terrestres tropicaux. Néanmoins, elles sont très peu étudiées et mal connues au Sénégal. Pour contribuer à combler cette lacune nous avons étudié le peuplement des fourmis de l'enclos d'acclimatation de Katané, un site se trouvant dans la Réserve de Faune du Ferlo Nord en zone de savane sahélienne (région de Matam, Sénégal). Les fourmis ont été échantillonnées à l'aide de pièges à fosse et par la chasse à vue. La richesse spécifique (S) est de 21 espèces. Les espèces les plus remarquables par leur abondance et leur distribution sont Crematogaster senegalensis, Monomorium areniphilum, Trichomyrmex abyssinicus, Monomorium bicolor, Pheidole andrieui, Brachyponera sennaarensis, Tetramorium angulinode papyri et Tetramorium sericeiventre. Elles appartiennent toutes à la sous-famille des Myrmicinae sauf $B$. sennaarensis qui est de la sous-famille des Ponerinae. L'indice de fourragement $(9,79)$, l'indice de Shannon $(0,95)$, l'indice d'équitabilité de Piélou $(0,77)$, l'indice de Simpson $(0,14)$ et l'indice de diversité de Simpson $(0,86)$ peuvent être utilisés comme référentiels pour le suivi de l'évolution des habitats de l'enclos et pour les études de l'état de dégradation des habitats dans le Ferlo en général.

() 2016 International Formulae Group. All rights reserved.

Mots clés: Fourmis, écosystèmes, diversité, abondance, Ferlo nord, Sénégal.

\section{Ants (Hymenoptera, Formicidae) of the acclimatization enclosure of Katane in the north Ferlo wildlife reserve (Senegal)}

\begin{abstract}
Ants (Hymenoptera, Formicidae), by their biomass and species diversity, play a fundamental role in the terrestrial ecosystems of the tropical area. Nevertheless, they are very little studied and poorly known in Senegal. To fill this gap we have chosen to study them in the acclimatization enclosure of Katane in the north Ferlo Reserve Wildlife situated in the Sahel savanna in Matam region (Senegal). They were sampled using pitfall traps and hand collecting. Species richness (S) is 21 species. The most remarkable for their abundances and distributions are Crematogaster senegalensis, Monomorium areniphilum, Trichomyrmex abyssinicus, Monomorium bicolor, Pheidole andrieui, Brachyponera sennaarensis, Tetramorium angulinode papyri and
\end{abstract}


Tetramorium sericeiventre. They all belong to the subfamily Myrmicinae except $B$. sennaarensis which belongs to the subfamily Ponerinae. The foraging index (9.79), the Shannon index (0.95), the index of Pielou equitability (0.77), the Simpson index (0.14) and the Simpson's index diversity (0.86) can be used as reference for monitoring the biotopes evolution in the enclosure of Katané and for the studies of land degradation in the Ferlo in general.

(C) 2016 International Formulae Group. All rights reserved.

Keywords: Ants, ecosystems, diversity, abundance, northern Ferlo, Senegal.

\section{INTRODUCTION}

Les Fourmis, parmi les plus communs et les plus diversifiés des insectes sociaux (Passera et Aron, 2005), sont les plus importantes en termes de biomasse (Hölldobler et Wilson, 1990 ; Passera et Aron, 2005). D'après Agosti et Johnson (2005), les Formicidae comptent plus de 12500 espèces qui peuvent présentées, à l'échelle locale, des assemblages diversifiés sous l'influence du climat, de l'altitude et de la qualité des habitats (Kaiser, 2014).

En Afrique, certaines espèces sont très actives dans la dégradation de la matière organique particulièrement pendant la saison sèche (Diop et al., 2013; Rouland et al., 2003). Parmi la macrofaune du sol la mieux adaptée aux régions arides (Kaiser, 2014), les Fourmis forment l'un des groupes clé de la dynamique et $\mathrm{du}$ fonctionnement des écosystèmes (Jones et al., 1994; Evans, 2011). Elles remanient d'importantes quantités de sol, à l'image des termites et des verres de terres; ce qui leur a valu la dénomination «ingénieurs de l'écosystème » par Jones et al. (1994).

Certaines Fourmis ont une activité fourragère sur les végétaux (Mony et al., 2009). Au niveau des réseaux trophiques, les fourmis sont les plus grands prédateurs d'arthropodes (Dyer, 2002 ; Philpott et al., 2008). De par cette fonction de prédation, elles constituent de bons agents de lutte biologique contre les bioagresseurs phytophages (Yemeda et al., 2013; Vayssieres et al., 2011).

Les Fourmis font l'objet d'un suivi dans l'étude des perturbations des écosystèmes terrestres (Bachelier, 1963 ; Lee et Foster, 1991). En effet, du fait du caractère sessile des colonies de la plupart des espèces et de leur sensibilité aux changements des conditions environnementales, elles sont de robustes bioindicateurs de la qualité des milieux. Elles sont utilisées dans de nombreux programmes d'évaluation de la biodiversité (Agosti et al., 2000).

Malgré ces fonctions multiples et importantes, les Fourmis restent encore mal connues. D'après Bolton et al. (2007), il existerait autant d'espèces de Fourmis connues que d'espèces à découvrir, en particulier dans les régions tropicales. Pour le cas du Sénégal, à notre connaissance, les seuls travaux réalisés sur la faune myrmécologique, sont ceux de Ndiaye (2010) et de Diamé et al. (2015).

Compte tenu de l'importance des Fourmis et le peu d'intérêt qui leur est accordé jusque là au Sénégal, nous nous sommes intéressés, dans un premier temps, au peuplement de Fourmis de l'enclos d'acclimatation de Katané, un site mis en défens dans la réserve de faune du Ferlo Nord.

\section{MATERIEL ET METHODES \\ Zone d'étude}

L'enclos d'acclimatation de Katané $\left(15^{\circ} 17^{\prime} 531\right.$ et $\left.13^{\circ} 57^{\prime} 508\right)$ est situé dans la réserve de faune du Ferlo nord (Figure 1). Le Ferlo nord appartient au domaine sahélien caractérisé par une saison des pluies de 3 à 4 mois allant de juillet à octobre. Le cumul de pluies enregistrées à la station de Ranérou en 2009 est de 710,6 mm d'après les données de l'Agence nationale de la météorologie du Sénégal (ANAMS). L'humidité relative atteint son maximum pendant la saison des pluies. Le 
mois de janvier est uniformément le mois le plus froid $\left(14,69{ }^{\circ} \mathrm{C}\right)$, le mois de mai $(43,91$ ${ }^{\circ} \mathrm{C}$ ) est généralement le mois le plus chaud (Source ANAMS, année 2009). Le paysage de type steppe et savane arbustive (Michel et al., 1969) est caractérisé par un tapis herbacé très riche $(\mathrm{Ba}, 2003)$ et une strate ligneuse dominée par des espèces comme Bombax costatum, Pterocarpus erinaceus, Combretum glutinosum, Acacia senegal, Balanites aegyptiaca et Boscia senegalensis. Les sols, de type ferrugineux tropicaux non lessivés appelés dior sont portés par un relief plat (Michel, 1965).

\section{Méthodes d'échantillonnages}

L'échantillonnage a été réalisé au mois de septembre 2010, pendant la saison des pluies. Les stations étudiées ont été choisies en fonction de la topographie, de la végétation et du type de sol. En tout, 6 stations ont été prospectées. Pour l'échantillonnage, nous avons utilisé la méthode des pièges à fosse (Pitfall traps) et la chasse à vue.

\section{Pièges à fosse}

Les pièges à fosse sont des pièges d'interception constitués par des pots en plastique de $7,5 \mathrm{~cm}$ de diamètre et $8,5 \mathrm{~cm}$ de profondeur (Figure 2). Les pots, contenant un fond d'eau savonneuse permettant de retenir les fourmis tombées dedans, sont disposés le long d'un transect avec un espacement de 10 $\mathrm{m}$.

Dans chaque station, 20 pièges sont posés, le même jour, le long d'un même transect. Ils sont relevés le lendemain à la même heure. Les fourmis capturées sont comptées puis conservées dans des tubes contenant de l'éthanol $70 \%$ avec une étiquette portant la date, le lieu, la station et le numéro du piège.

\section{Chasse à vue}

$\mathrm{Au}$ niveau de chaque station, les fourmis sont échantillonnées dans une aire de $100 \mathrm{~m}$ x $20 \mathrm{~m}$. Elles sont recherchées dans les fourmilières, la litière, sur le bois mort, les arbres, dans le sol, sous placages des termites et dans les termitières... Les Fourmis capturées à l'aide de pinces souples sont conservées dans des tubes contenant de l'éthanol $70 \%$ avec une étiquette portant la date, le lieu, la station, le micro-habitat (arbre, sol, bois mort, pied d'arbre, fourmilière, termitière, ou placage), le numéro de référence ainsi que le nom du récolteur.

\section{Identification des espèces}

Les Fourmis récoltées ont été identifiées grâce à l'ouvrage de Hölldobler et Wilson (1990) et à Ants of Africa, une base en ligne de B. Taylor (Taylor, 2015). Les spécimens qui n'ont pu être identifiés par ces moyens ont été envoyés à B. Taylor.

\section{Caractérisation des peuplements \\ La richesse $(S)$}

La richesse spécifique correspond au nombre d'espèces rencontrées dans les différentes stations du site d'étude. Elle est obtenue après identification de l'ensemble des spécimens récoltés par la chasse à vue ou pris dans les pièges.

\section{L'Abondance (A)}

L'abondance (A) d'une espèce correspond au nombre total d'individus de l'espèce. Elle ne s'applique qu'aux espèces capturées par la méthode des pièges à fosse permettant de faire un dénombrement. L'abondance est complétée par le taux de fourragement qui est le nombre moyen d'individus capturés par piège (Chazeau et al., 2003).

\section{L'Indice de Shannon (H') et indice d'équitabilité de Piélou (J')}

L'indice de Shannon (H') est obtenu par la formule

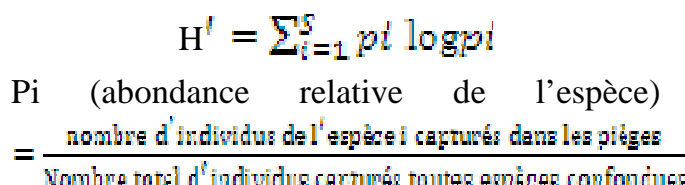

Le logarithme base 2 est ici utilisé.

L'indice d'équitabilité de Piélou (J') est calculé par la formule : $I^{\prime}=\frac{\mathrm{H}^{\prime}}{\mathrm{H}^{\prime} \max }$ $\mathrm{H}_{\text {max }}=\log \mathrm{S}$ (S correspond ici au nombre d'espèces issues de l'identification des spécimens pris dans les pièges à fosse. 


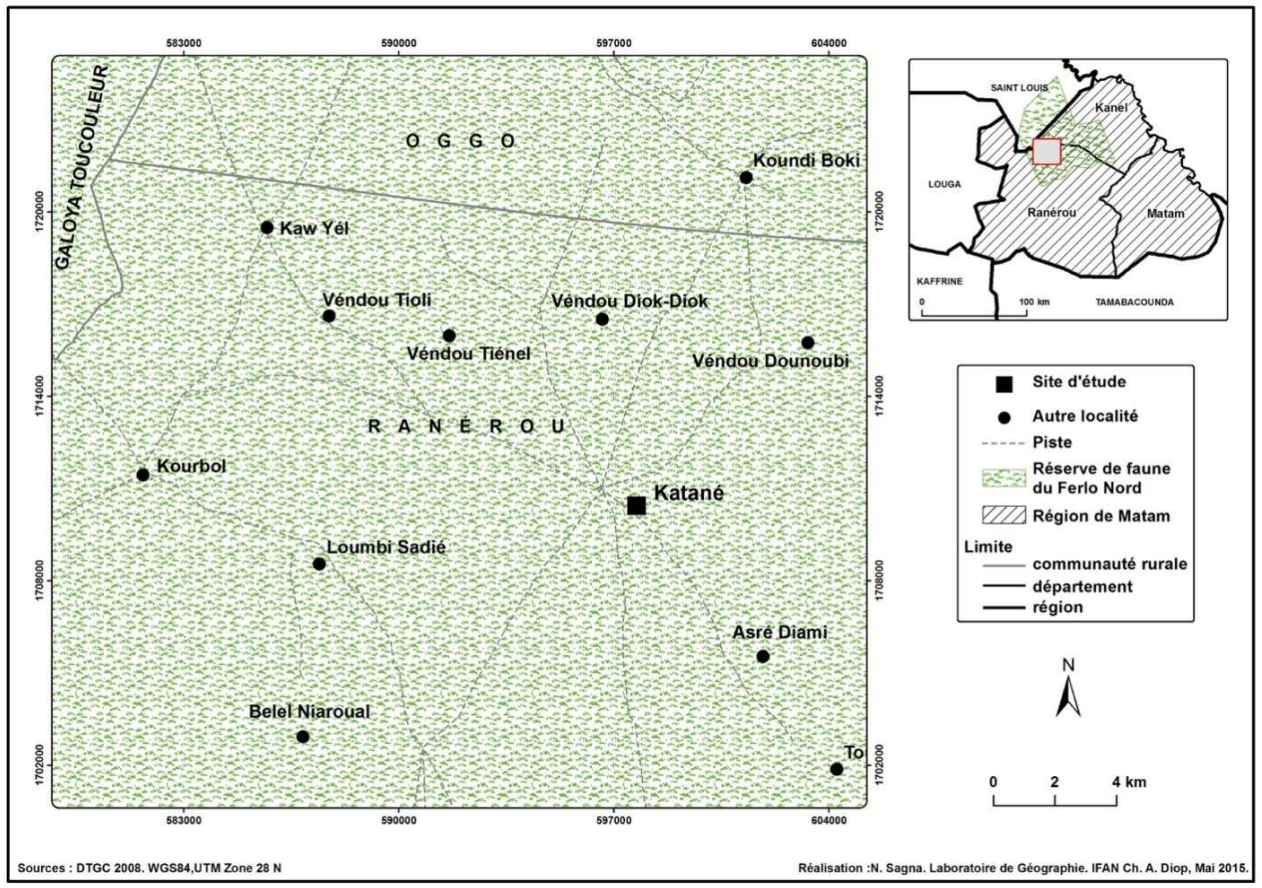

Figure 1 : Localisation du site de Katané dans la région Nord du Sénégal.

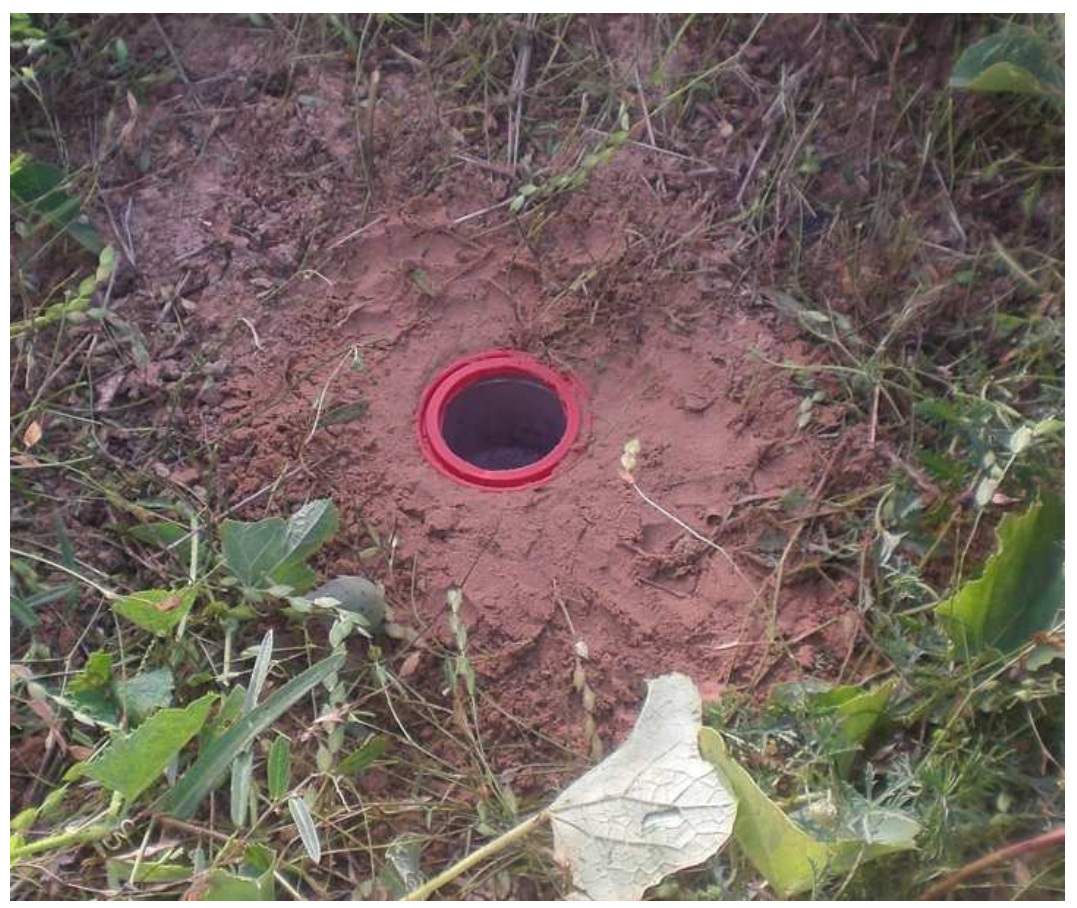

Figure 2 : Piège à fosse. 
Indice de Simpson ( $L$ ) et indice de diversité de Simpson $(D)$

L'indice de Simpson (L) est calculé selon la formule :

$$
\begin{gathered}
\mathrm{L}=\sum[\mathrm{ni}(\mathrm{ni}-1)] /[\mathrm{N}(\mathrm{N}-1)] \\
\mathrm{ni}={ }_{\text {nombre d'individus de } \mathrm{l}^{\prime} \text { espèce } \mathrm{i} \text { capturès dans les pièges }} \\
\mathrm{N}=\text { Nombre total d'individus capturés toutes espèces confondues }
\end{gathered}
$$

L’indice de diversité de Simpson est calculé par la formule :

$$
\mathrm{D}=1-\mathrm{L}=\sum\{[\text { ni(ni-1) }] /[\mathrm{N}(\mathrm{N}-1)]\}
$$

\section{RESULTATS}

\section{Inventaire des espèces}

La richesse spécifique (S) des Fourmis de l'enclos de Katané est de 21 espèces réparties dans 12 genres et 4 Sous-familles que sont les Cerapachyinae, les Ponerinae, les Myrmicinae et les Formicinae.

La sous-famille des Myrmicinae, avec 7 genres (Crematogaster, Meranoplus, Monomorium Myrmicaria, Pheidole, Tetramorium et Trichomyrmex) et 12 espèces, est la plus diversifiée. Les Formicinae comptent 3 genres (Camponotus, Lepisota et Polyrachis) et 7 espèces. Quand à la sousfamille des Ponerinae, elle est la moins diversifiée avec 1 genre (Brachyponera) et 1 espèce (Brachyponera sennaarensis). La sousfamille des Cerapachyinae est aussi représentée par 1 seul genre (Cerapachys) et 1 espèce, Cerapachys cribrinodis (Tableau 1).

Les espèces Cerapachys cribrinodis, Monomorium balathir, Lepisiota gracilicornis et Polyrhachis viscosa, ont été uniquement récoltées par la chasse à vue alors que les espèces Myrmicaria distincta et Trichomyrmex oscaris ont été capturées exclusivement à l'aide des pièges à fosse.

\section{Caractérisation du peuplement des}

Fourmis

Abondance des espèces de fourmis à l'échelle de l'enclos

L'abondance moyenne des fourmis à l'échelle de l'enclos est de 9,8 individus par piège. En fonction des espèces, l'abondance moyenne des fourmis capturées est variable (Figure 3). L'analyse de la variance (ANOVA, seuil 5\%), permet de répartir les espèces en 3 groupes entre lesquels les différences observées sont statistiquement significatives. Le premier groupe comprend une espèce, Monomorium bicolor, qui domine les autres espèces par son abondance. Le deuxième groupe, intermédiaire, comprend deux espèces, Pheidole andrieui et Tetramorium sericeiventre. Le troisième groupe comprend 14 espèces. Il est à noter qu'à l'intérieur de ce dernier groupe, certaines espèces ont des abondances significativement différentes entre elles. Les espèces de ce groupe sont les moins abondantes.

\section{Abondance des fourmis à l'échelle des stations}

A l'échelle des stations, on observe une certaine variation de l'abondance des fourmis (Figure 4). Une analyse de la variance (Anova, seuil de 5\%) de l'abondance des fourmis, toutes espèces confondues, permet de classer les stations en deux groupes. Les fourmis sont plus abondantes dans les stations 1 et 5 (premier groupe) que dans les stations $2,3,4$ et 6 (deuxième groupe) où les captures sont significativement plus faibles.

\section{Diversité des fourmis \\ Indice de diversité de Shannon et indice d'équitabilité de Piélou}

L'indice de Shannon H' est égal à 0,95. Cet indice tend vers H'max $(1,23)$ et confirme ainsi l'existence de plusieurs espèces ayant la même abondance. Quant à la répartition des espèces, l'indice de Piélou $(0,77)$ proche de 1 , montre qu'il y a une certaine équitabilité des espèces par leurs effectifs.

\section{Indice de Simpson et l'indice de diversité de Simpson}

Concernant la diversité des espèces, l'indice de la valeur mesurée de Simpson $(0,14)$ indique une faible diversité des espèces de fourmis dans l'enclos de Katané. Quant à l'indice de diversité de Simpson $(0,86)$, il traduit là également la codominance de plusieurs espèces. 


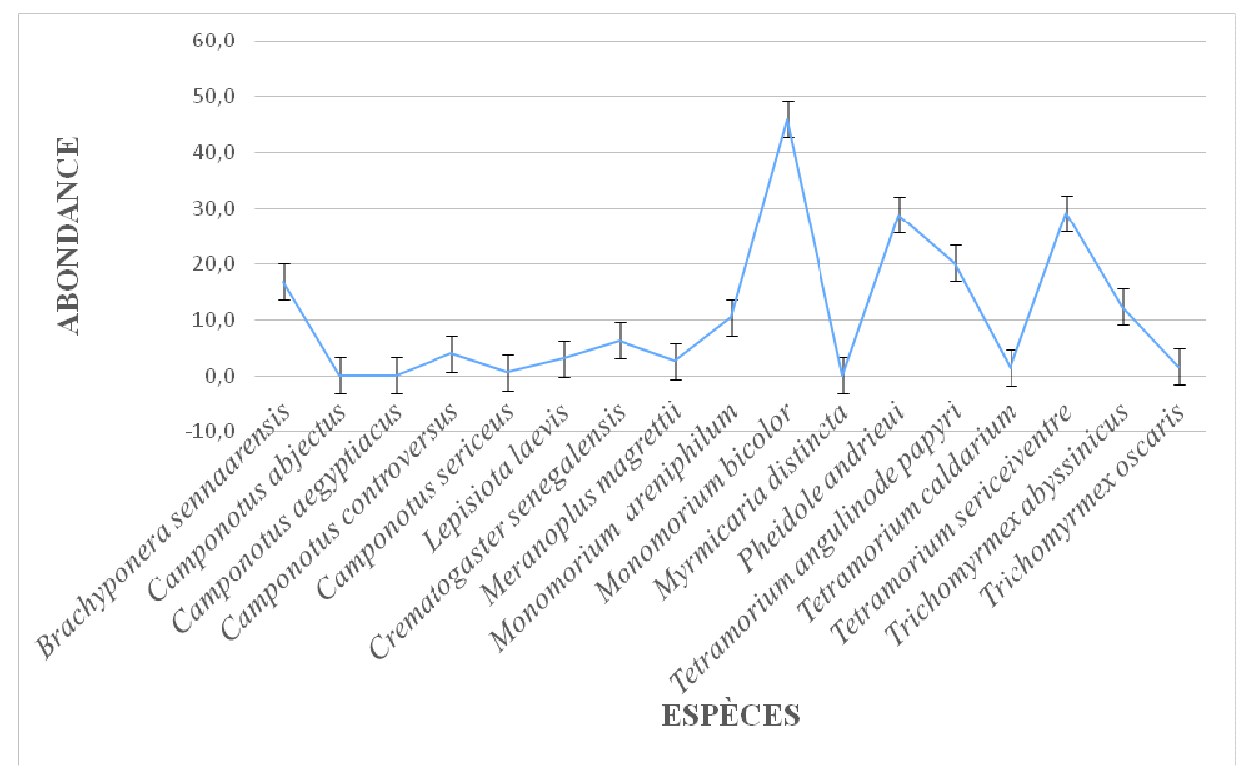

Figure 3 : Abondance des espèces de fourmis à l'échelle de l'enclos.

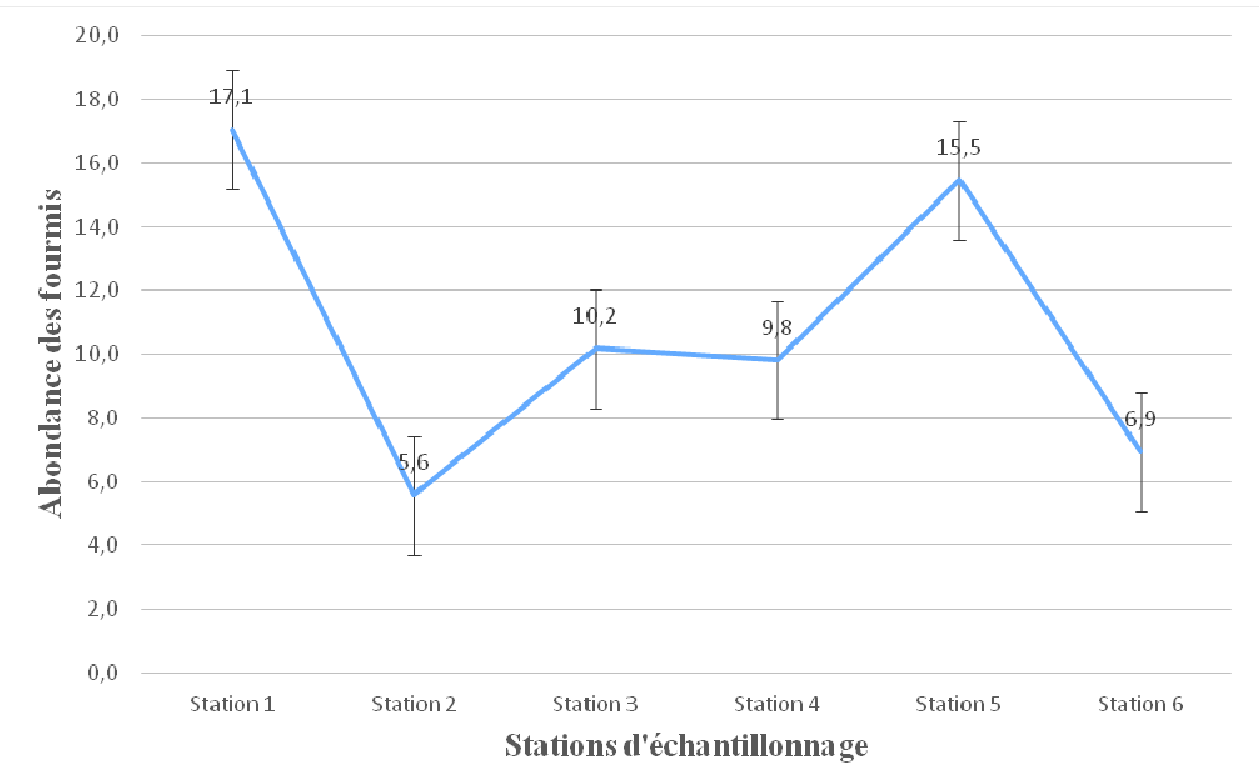

Figure 4 : Abondance des fourmis à l'échelle des stations. 
Tableau 1 : Liste des espèces de fourmis rencontrées dans l'enclos d'acclimations de Katané (Matam, Sénégal).

\begin{tabular}{|c|c|c|c|}
\hline Sous-famille & Genre & Espèces & Échantillonnage \\
\hline $\begin{array}{l}\text { CERAPACHYINAE } \\
\text { Wheeler }\end{array}$ & $\begin{array}{l}\text { Cerapachys } \\
\text { Smith }\end{array}$ & $\begin{array}{ll}\text { Cerapachys } & \text { cribrinodis } \\
\text { Weber, } 1942 & \end{array}$ & Chasse à vue \\
\hline $\begin{array}{l}\text { PONERINAE (Lepeletier } \\
\text { de Saint-Fargeau) }\end{array}$ & $\begin{array}{l}\text { Brachyponera } \\
\text { Emery }\end{array}$ & $\begin{array}{l}\text { Brachyponera } \\
\text { sennaarensis } \\
\text { 1862) }\end{array}$ & $\begin{array}{l}\text { Piège à fosse, chasse } \\
\text { à vue }\end{array}$ \\
\hline \multirow{12}{*}{$\begin{array}{l}\text { MYRMICINAE } \\
\text { (Lepeletier de Saint- } \\
\text { Fargeau) }\end{array}$} & $\begin{array}{l}\text { Crematogaster } \\
\text { Lund }\end{array}$ & $\begin{array}{l}\text { Crematogaster } \quad(C r .) \\
\text { senegalensis } \text { Roger, } 1863\end{array}$ & $\begin{array}{l}\text { Piège à fosse, chasse } \\
\text { à vue }\end{array}$ \\
\hline & $\begin{array}{l}\text { Meranoplus } \\
\text { Smith F. }\end{array}$ & $\begin{array}{ll}\text { Meranoplus } & \text { magrettii } \\
\text { André, } 1884 & \end{array}$ & $\begin{array}{l}\text { Piège à fosse, chasse } \\
\text { à vue }\end{array}$ \\
\hline & \multirow{3}{*}{$\begin{array}{l}\text { Monomorium } \\
\text { Mayr }\end{array}$} & $\begin{array}{l}\text { Monomorium areniphilum } \\
\text { Santschi, } 1911\end{array}$ & $\begin{array}{l}\text { Piège à fosse, chasse } \\
\text { à vue }\end{array}$ \\
\hline & & $\begin{array}{l}\text { Monomorium } \\
\text { Emery, } 1877\end{array}$ & $\begin{array}{l}\text { Piège à fosse, chasse } \\
\text { à vue }\end{array}$ \\
\hline & & $\begin{array}{l}\begin{array}{l}\text { Monomorium } \\
\text { (Bolton, 1987) }\end{array} \quad \text { balathir }\end{array}$ & Chasse à vue \\
\hline & $\begin{array}{l}\text { Myrmicaria } \\
\text { Saunders }\end{array}$ & $\begin{array}{ll}\text { Myrmicaria } & \text { distincta } \\
\text { Santschi, 1925 } & \end{array}$ & Piège à fosse \\
\hline & $\begin{array}{l}\text { Pheidole } \\
\text { Westwood }\end{array}$ & $\begin{array}{l}\text { Pheidole andrieui } \\
\text { Santschi, } 1930\end{array}$ & $\begin{array}{l}\text { Piège à fosse, chasse } \\
\text { à vue }\end{array}$ \\
\hline & \multirow{3}{*}{$\begin{array}{l}\text { Tetramorium } \\
\text { Mayr }\end{array}$} & $\begin{array}{ll}\text { Tetramorium } & \text { caldarium } \\
\text { (Roger, 1857) } & \\
\end{array}$ & $\begin{array}{l}\text { Piège à fosse, chasse } \\
\text { à vue }\end{array}$ \\
\hline & & $\begin{array}{l}\text { Tetramorium angulinode } \\
\text { papyri (Santchi, 1914) }\end{array}$ & $\begin{array}{l}\text { Piège à fosse, chasse } \\
\text { à vue }\end{array}$ \\
\hline & & $\begin{array}{l}\text { Tetramorium sericeiventre } \\
\text { Emery, } 1877\end{array}$ & $\begin{array}{l}\text { Piège à fosse, chasse } \\
\text { à vue }\end{array}$ \\
\hline & \multirow{2}{*}{$\begin{array}{l}\text { Trichomyrmex } \\
\text { Mayr }\end{array}$} & $\begin{array}{l}\text { Trichomyrmex abyssinicus } \\
\text { (Forel, 1894) }\end{array}$ & $\begin{array}{l}\text { Piège à fosse, chasse } \\
\text { à vue }\end{array}$ \\
\hline & & $\begin{array}{ll}\begin{array}{l}\text { Trichomyrmex } \\
\text { (Forel, 1894) }\end{array} & \\
\end{array}$ & Piège à fosse \\
\hline \multirow{7}{*}{ FORMICINAE Wheeler } & \multirow{4}{*}{$\begin{array}{l}\text { Camponotus } \\
\text { Mayr }\end{array}$} & $\begin{array}{ll}\text { Camponotus abjectus } \\
\text { Santschi, } 1937\end{array}$ & $\begin{array}{l}\text { Piège à fosse, chasse } \\
\text { à vue }\end{array}$ \\
\hline & & $\begin{array}{l}\text { Camponotus aegyptiacus } \\
\text { Emery, } 1915\end{array}$ & $\begin{array}{l}\text { Piège à fosse, chasse } \\
\text { à vue }\end{array}$ \\
\hline & & $\begin{array}{l}\text { Camponotus controversus } \\
\text { Viehmeyer, } 1914\end{array}$ & $\begin{array}{l}\text { Piège à fosse, chasse } \\
\text { à vue }\end{array}$ \\
\hline & & $\begin{array}{l}\text { Camponotus sericeus } \\
\text { (Fabricius, 1798) }\end{array}$ & $\begin{array}{l}\text { Piège à fosse, chasse } \\
\text { à vue }\end{array}$ \\
\hline & \multirow{2}{*}{ Lepisiota (Mayr) } & $\begin{array}{l}\text { Lepisiota gracilicornis } \\
\text { (Forel, 1892) }\end{array}$ & Chasse à vue \\
\hline & & $\begin{array}{l}\text { Lepisiota laevis (Santschi, } \\
\text { 1913) }\end{array}$ & $\begin{array}{l}\text { Piège à fosse et } \\
\text { chasse à vue }\end{array}$ \\
\hline & $\begin{array}{l}\text { Polyrhachis } \\
\text { Smith F. }\end{array}$ & $\begin{array}{l}\text { Polyrhachis viscosa Smith } \\
\text { F., } 1858\end{array}$ & Chasse à vue \\
\hline
\end{tabular}




\section{DISCUSSION}

La diversité spécifique observée dans l'enclos de Katané est de 21 espèces réparties dans 4 sous-familles. A l'échelle des stations, on note une répartition différente des espèces de fourmis malgré les dimensions relativement réduites du site étudié. Le lien étroit entre les habitats et les peuplements fait des Fourmis un groupe idéal dans l'évaluation de la biodiversité (Agosti et al., 2000).

C'est la première étude faunistique des Fourmis menée dans la réserve de faune du Ferlo nord. Une étude similaire réalisée par Ndiaye (2010) à Sabodala, localité située en zone soudanienne à l'est du Sénégal, avait révélé une richesse spécifique plus élevée avec 36 espèces. Les différences peuvent s'expliquer par des facteurs d'ordre climatique plus ou moins accentués par la période et le nombre de stations d'échantillonnage. En effet, Ndiaye (2010) a exploré un plus grand nombre de stations (14) et une plus grande diversité de biotopes (5) aussi bien en saison sèche qu'en saison des pluies. La richesse spécifique des fourmis dans des vergers à Thiès (Diame et al., 2015) plus importante, avec 49 espèces, 21 genres et 7 sous-familles, est, comme à Katané, prédominée par les Myrmicinae, particulièrement les genres Pheidole et Monomorium. Au niveau de la composition spécifique, on note que 8 espèces communes avec la faune de: Brachyponera sennaarensis Camponotus sericeus, Crematogaster senegalensis, Meranoplus magrettii, Monomorium areniphilum, Monomorium bicolor, Pheidole andrieui, Tetramorium sericeiventre.

Par rapport aux espèces antérieurement connues au Sénégal (Ndiaye, 2010; Diame et al., 2015), il est noté 7 espèces nouvelles pour le pays. Il s'agit de: Cerapachys cribrinodis, Monomorium balathir, Myrmicaria distincta, Tetramorium caldarium, Tetramorium angulinode papyri, Camponotus sericeus et Lepisiota gracilicornis.

Camponotus sericeus, connu $\mathrm{du}$ Soudan (Taylor, 2015) semble être une espèce inféodée à la savane sahélienne du Soudan au Sénégal.

Le genre Cerapachys nomade (Brady, 2003), encore appelé Yunodorylus (Borowiec,
2009) est panafricain (Wheeler et al., 1922 ; Urbani et al., 1992). Cependant, Cerapachys cribrinodis, espèces appartenant au sous-genre Parasyscia, jugé particulièrement rare en Afrique par Weber (1949), était connue du Cameroun, de la RD Congo et de la Guinée (Taylor, 2015). Avec la découverte de l'espèce au Sénégal, son aire de distribution s'en trouve élargie.

L'étude de l'abondance des espèces a montré une dominance des Myrmicinae avec notamment Monomorium bicolor, Pheidole andrieui et Tetramorium angulinode papyri et Trichomyrmex abyssinicus. Cette dernière espèce, qui a changé de genre (Ward et al., 2014), est très présente à travers toute la zone sahélienne. Quand à Monomorium areniphilum, elle est qualifiée d'espèce saharienne par Bolton (1987). Elle est connue au Mali, au Soudan, au Sénégal et au Niger (Taylor, 2015). Camponotus aegyptiacus et les espèces du genre Cataglyphis seraient typiques de la zone saharienne d'après Delye (1967).

D'après Lévieux (1972), la limite sud de l'aire de répartition des espèces du genre Myrmicaria spp. et de celle de Tetramorium sericeiventre, se situerait dans les savanes nord de Côte d'Ivoire où leurs densités sont faibles. Concernant Tetramorium sericeiventre, Taylor (2015) note qu'elle est signalée en Afrique de l'Est (Érythrée, Tanzanie, Somalie, Éthiopie, Kenya Ouganda, Zambie Soudan), en Afrique du Sud (Zimbabwe Botswana, Lésotho, Malawi, Namibie, Mozambique, Afrique du Sud) et en Afrique centrale (Gabon, Congo, en Centre Afrique RD Congo, Cameroun, Angola) et en Afrique de l'Ouest (Nigéria, Guinée, Burkina Faso, Ghana, Libéria, Mali, Gambie). La présence de l'espèce en zone sahélienne aride du Sénégal confirme la tendance panafricaine de sa distribution. Pour ce qui est de Myrmicaria distincta, sa rareté à Katané est conforme à son statut d'espèce à affinité soudano-guinnéenne marquée (Ndiaye, 2010).

L'abondance moyenne de fourmis capturées par piège et par jour, toutes espèces 
confondues est de 9,79 individus. Au niveau des stations, cette moyenne varie de 5,28 à 15,26. Cette abondance relative des fourmis dans un milieu aride s'explique par la bonne adaptation de certaines espèces, les Myrmicinae et Brachyponera sennareensis notamment, à ce type de milieu. En effet, comme l'a souligné Donzé (2011), le succès écologique des Fourmis, au-delà de la vie sociale, est lié à leur capacité d'adaptation hors norme, en tant que groupe, à des milieux très diversifiés. La présence remarquable des Termites (Guèye, 2011) et la permanence du tapis graminéen et l'action anthropique limité sur le site sont autant facteurs favorables aux fourmis. Cette abondance moyenne des fourmis, appelée indice de fourragement (Chazeau et al., 2003), permet d'évaluer le degré de dégradation des habitats.

Le profil myrmécologique de l'enclos est caractéristique des zones arides. En effet, d'après Lévieux (1972) les peuplements de fourmis des zones forestières se caractérisent par une dominance des Ponerinae. A Sabodala, savane soudanienne du Sénégal oriental, d'après les travaux de Ndiaye (2010), la faune myrmécologique montre une diversité des Ponerinae plus importante qu'à Katané (zone sahélienne) mais moins importante qu'en savane guinéenne de la Guinée (Bernard, 1952) et de la Côte d'Ivoire (Lévieux, 1972).

\section{Conclusion}

$\mathrm{Au}$ terme de cette étude préliminaire sur les fourmis de la réserve de faune du Ferlo nord, il apparait que la faune myrmécologique de Katané, caractéristique des zones tropicales sèches, est dominée par les Myrmicinae avec Monomorium bicolor, Pheidole andrieui et Tetramorium angulinode papyri. L'étude a permis de relever la présence de 7 nouvelles espèces pour le Sénégal. Il s'agit de Cerapachys cribrinodis, de Monomorium balathir, de Myrmicaria distincta, de Tetramorium caldarium, de Tetramorium angulinode papyri, de Camponotus sericeus, et de Lepisiota gracilicornis). Le profil myrmécologique, l'indice de fourragement, l'indice de Shannon et l'indice de Simpson pourraient servir de référentiels pour le suivi de l'évolution du milieu.

\section{CONFLIT D'INTERETS}

Les auteurs déclarent qu'il n'y a aucun conflit d'intérêts pour cet article.

\section{CONTRIBUTIONS DES AUTEURS}

MMD est l'investigateur principal ; il a participé à la conception du protocole, a réalisé l'échantillonnage et l'identification, et a rédigé le manuscrit. $\mathrm{ABN}$ et $\mathrm{ChTB}$ ont conçu le protocole et supervisé les travaux de recherche. BT a contribué à l'identification et à la rédaction du manuscrit.

\section{REFERENCES}

Agosti D, Johnson NF. 2005. antbase.org. http://www.antbase.org/ants/africa/

Agosti D, Majer JD, Alonso LE, Schultz TR. 2000. Ants. Standard Methods for Measuring and Monitoring Biodiversity. Smithsonian Institution Press : Washington and London, 1-59.

Ba DM. 2003. Second Séminaire sur les Antilopes Sahélo-Sahariennes, Rapport National du Sénégal. Proceedings du second séminaire régional sur la conservation et la restauration des antilopes sahélo-saharienne. Agadir, 1-5 mai 2003, CMS Technical Series Publication, 150-155.

Bachelier G. 1963. La Vie dans les Sols. ORSTOM: Paris; 480 p.

Bernard F. 1952. La réserve naturelle intégrale du $\mathrm{M}^{\mathrm{t}}$ Nimba. Fascicule $\mathrm{I}$. Mémoires de l'Institut Fondamental d'Afrique Noire, 19 : 165-269.

Bolton B. 1987. A review of the Solenopsis genus-group and revision of Afrotropical monomorium Mayr (Hymenoptera: Formicidae). Bulletin of the British Museum (natural history). Entomology series, 54 (3): 1-192.

Bolton B, Alpert G, Ward PS, Naskrecki P. 2007. Bolton's Catalogue of Ants of the 
World 17582005. Harvard University Press: Cambridge, MA, USA, CD-ROM.

Borowiec ML. 2009. New ant species related to Cerapachys sexspinus and discussion of the status of Yunodorylus (Hymenoptera: Formicidae). Zootaxa, 2069: $\quad 43-58 . \quad$ DOI: http://www.mapress.com/zootaxa/list/20 09/zt02069.html.

Brady SG. 2003. Evolution of the army ant syndrome: The origin and long-term evolutionary stasis of a complex of behavioral and reproductive adaptations. PNAS, 100(11): 6575-6579. DOI : http://www.pnas.org/content/100/11/657 5.full.

Chazeau J, Jourdan H, Bonnet de Larbogne L, Konghouleux J, Potiaroa T. 2003. Recherche des caractéristiques faunistiques des habitats se trouvant sur les sites retenus pour l'installation des infrastructures minières et industrielles du complexe de Goro Nickel. Rapport préliminaire. DOI : www.oeil.nc/cdrn/index.php/files/biblio graphie/243.

Delye G. 1967. Physiologie et Comportement de quelques Fourmis. (Hymenoptère, Formicidae), du Sahara en Rapport avec les Principaux Facteurs du Climat. Insectes Sociaux, 14(4) : 323-338.

Diame L, Blatrix R, Grechi I, Rey J-Y, Sane CAB, Vayssieres J-F, De Bon H, Diarra K. 2015. Relations between the design and management of Senegalese orchards and ant diversity and community composition. Agriculture, Ecosystems and Environment, 212: 94-105. DOI: http://dx.doi.org/10.1016/j.agee.2015.07. 004

Diop A, Ndiaye AB, Ba CT. 2013. Décomposition de la bouse de bovin sèche et macrofaune associée en zone sahélienne semi-aride (Matam, Sénégal). Int. J. Biol. Chem. Sci., 7(1) : 147-162. DOI : http://dxdoi.org/104314/ijbcs.v7i1.12
Donzé A. 2011. Approche expérimentale et théorique de l'écologie des fourmis des bois. Bulletin de la Société des Enseignants Neuchâtelois de Sciences, 2(40) : 1-20. DOI : www.sensneuchatel.ch/bulletin/no40/art2-40donze.pdf

Dyer LA. 2002. A quantification of predation rates, indirect positive effects on plants, and foraging variation of the giant tropical ant, Paraponera clavata. Journal of Insect Science, 2(18): 1-7. DOI: insectscience.org/2.18

Evans TA, Dawes TZ, Ward PR, Lo N. 2011. Ants and termites increase crop yield in a dry climate. Nat Commun, 2: 262. DOI: $10.1038 /$ ncomms1257.

Gobat JM, Aragno M, Matthey W. 2010. Le Sol vivant. Bases de pédologie-Biologie des sols. (3 ${ }^{\text {ème }}$ ed.). Presses Polytechniques et Universitaires Romandes : Lausannes ; 817 p.

Guèye S. 2011. Contribution à l'étude de la faune de Termites (Isoptera) dans la réserve de faune du Ferlo Nord (Sénégal): cas de l'enclos d'acclimatation de Katané. Mémoire de Master, UCAD, 28 p.

Hölldobler B, Wilson EO. 1990. The Ants. The Belknap Press of Havard University Press Cambridge, Massachusetts; 1-3.

Jones CG, Lawton JH, Shachak M. 1994. Organisms as ecosystem engineers. Ecosystem Management. Oikos: New York; 373-386.

Kaiser DT. 2014. Termites and ants in Burkina Faso (West Africa) - taxonomic and functional diversity along land-use gradients - ecosystem services of termites in the traditional zaï system. Dissertation zur Erlangung des naturwissenschaftlichen Doktorgrades der Julius-Maximilians-Universität, Würzburg, p.274.

Lee K, Foster RC. 1991. Soil fauna and soil structure. Aust. J. Soil Res., 29 : 745-75.

Lévieux J. 1972. Les Fourmis de la savane de Lamto (Côte d'Ivoire): éléments de 
taxonomie. Bulletin de l'Institut Fondamental d'Afrique Noire, série A, 34(3) : 611-654.

Michel P. 1965. Pédologie. In Atlas National du Sénégal. Presses de l'Institut Géographique National : Paris, 40-41.

Michel P, Naegele A, Toupet C. 1969. Contribution à l'étude biologique du Sénégal septentrional. 1. Le milieu naturel. Bulletin de l'Institut Fondamental d'Afrique Noire, série A, 31(3-4) : 756-839.

Mony R, Ondoya J, Dibong S, Issaka J, Akoa A. 2009. Myrmécofaune arboricole associée aux couples Phragmenthera capitata (Sprengel) S.Ballel hôte au verger de la chefferie de Ndogbong (Douala, Cameroun). Int. J. Biol. Chem. Sci., 3(6): 1991-8631. DOI: http://dx.doi.org/10.4314/ijbcs.v3i6.5315 5.

Ndiaye AB. 2010. Etude de la faune entomologique de la concession d'Oromin à Sabodola (Sénégal). Rapport final, $24 \mathrm{p}$.

Passera L, Aron S. 2005. Les Fourmis : Comportement, Organisation Sociale et Evolution. Les Presses Scientifiques du CNRC: Ottawa; 1-24.

Philpott SM, Perfecto I, Vandermeer J. 2008. Effects of predatory ants on lower trophic levels across a gradient of coffee management complexity. Journal of Animal Ecology, 77(3): 505-511. DOI: 10.1111/j.1365-2656.2008.01358.x

Rouland C, Lepage M, Chotte JL, Diouf M, Ndiaye D, Ndiaye S, Seuge C, Brauman A. 2003. Experimental manipulation of termites (Isoptera, Macrotermitinae) foraging patterns in a Sahelo-Sudanese savanna: effect of litter quality. Insectes Sociaux, 50(4): 309-316. DOI: 10.1007/s00040-003-0680-6
Urbani CB, Bolton B. Ward PS. 1992. The internal phylogeny of ants (Hymenoptera Formicidae). Systematic Entomology, 17: 301-329.

Vayssieres F, Sinzogan A, Korie S, Adandonon A, Worou S. 2011. Field observational studies on circadian activity pattern of Oecophylla longinoda (Latreille) (Hymenoptera: Formicidae) in relation to abiotic factors and mango cultivars. Int. J. Biol. Chem. Sci., 5(2): 790-802.

DOI: http://dx.doi.org/10.4314/ijbcs.v5i2.7215 3

Ward PS, Brady SG, Fisher BL, Schultz TR.2014. The evolution of myrmicine ants: phylogeny and biogeography of a hyperdiverse ant clade (Hymenoptera: Formicidae). Systematic Entomology, 40(1): 61-81. DOI: 10.1111/syen.12090

Weber NA. 1949. New African ants of the genera Cerapachys, Phryacaces, and Simopon. American Museum Novitates, 1396: $1-9$. DOI: http://hdl.handle.net/2246/4409

Wheeler WM, Bequaert J, Bailey IW, Santchi F, Mann WM. 1922. Ants of the American Museum Congo Expedition. A contribution to the myrmecology of Africa of natural History. 1. Bulletin of the American Museum of Natural History, XLV: 52-1005.

Yemeda CFL, Mony R, Tchatat M, Dibong S. 2013. Contribution des fourmis à la lutte biologique contre les Loranthacea. Int. J. Biol. Chem. Sci., 7(3): 924-937. DOI : http://dx.doi.org/104314/ijbcs.v7i3.4

Taylor B. 2015. The Ants of (sub-Saharan) Africa. http://www.antsofafrica.org/ 